\title{
Optoacoustic Measurements for Small-Volume Liquid Samples
}

\author{
S. EGEREV* \\ Andreyev Acoustics Institute, 4 Shvernika str., Moscow 117036, Russia
}

\begin{abstract}
High sensitivity of optoacoustic measurement method makes it possible to use it for the analysis of the liquid samples having microliter volume. The core of any measurement optoacoustic system is represented by a so-called optoacoustic cell. The paper deals with a layered prism optoacoustic cell. It is well-suited for the small-volume sample analysis. In the paper, laser-induced elastic pulse in the layered prism cell is examined at the stage of its generation, at the stage of its propagation through the body of the cell, and at the stage of the wideband pick-up with the piezoelectric transducer. This pulse serves to determine trace concentrations of various impurities in the sample. The optimization of layered prism cell parameters is proposed. Figures of merit of optoacoustic determination of lipopolysaccharides in the liquid small-volume sample with the use of the optimized layered prism cell are evaluated as an example.
\end{abstract}

DOI: $10.12693 /$ APhysPolA.127.10

PACS: 43.35.Bf, 43.35.Sx, 05.70.-a, 42.40.My, 42.55.-f

\section{Introduction}

Optoacoustic $(\mathrm{OA})$ calorimetric measurement method is actively used in chemistry as a very sensitive technique of molecular absorption spectroscopy [1,2]. The calorimetric measurement of this kind is based on the pulse laser-induced thermal optoacoustic conversion in a small volume of a liquid sample. Very informative acoustic response thus radiated by the sample serves for the measurements of the sample's parameters. The figures of merit of the $\mathrm{OA}$ measurement method applied for relatively large-volume liquid samples were considered in $[2,3]$.

Nowadays, the chemical analysis is conducted in the samples of a very small volume, order of units and tens of microliters [4]. This is to meet the requirements of pharmaceutics, biology and medicine. The high sensitivity of OA calorimetry makes it possible to use it for the analysis of the liquid samples having microliter volume.

However, OA measurements applied to the liquid small-volume samples have some peculiarities. The important factor here is the influence of the boundaries of the sample upon the profile of the elastic response. This makes the interpretation of the signal somewhat difficult. We consider sound generation by the laser pulse in a small (microliter) volume liquid sample. The sample consists of both solvent and solute. In turn, the solute is characterized by some unknown trace molar concentration $C$. The elastic signal outgoing from the sample should serve to evaluate $C$. To obtain the parameters of an outgoing elastic signal one should estimate an instantaneous pressure increment in a sample following the non-radiative relaxation in the laser-heated small-volume sample. This can be done within the framework of an initial-value problem and using the adiabatic approach [4] as

*e-mail: segerev@gmail.com

$$
p_{0}=\frac{\alpha \beta c^{2} E_{0}}{\pi a^{2} C_{p}}
$$

where $\alpha$ is the total sample absorptivity, $E_{0}$ is the laser pulse energy $\left(E_{0} \approx \pi a^{2} I_{0} \tau\right), \tau$ is the laser pulse width, $I_{0}$ is peak intensity, $a$ is the beam cross-section radius, $\beta$ is the volume thermal expansion coefficient, $c$ is the sound velocity in the sample, $C_{p}$ is the specific heat. This increment propagates as an outgoing elastic pulse and can be recorded by a transducer at some distance from the sample.

The energy density released in the sample is very small. Hence, the thermal expansion of the sample which gives rise to the acoustic outgoing radiation is of linear type. The sample is considered to be optically "thin", $\alpha L \ll 1$, where $L$ is a thickness of the sample, close to the laser beam travel path. For the case of a short laser pulse a specific ratio between the characteristic lengths of the problem takes place. It is correctly reasoned that $L>$ $d_{\mathrm{ac}} \gg d_{\mathrm{th}}$, where $d_{\mathrm{ac}}=c \tau$ is a sound travel path during the release of optical energy and $d_{\mathrm{th}}$ is the scale of the diffusion of the heat. In this case, adiabatic approach to the approximate solution of our initial-value problem is correct. For example, for typical cases, $d_{\mathrm{ac}}$ is about $5 \times 10^{-5} \mathrm{~m}$, while $d_{\mathrm{th}}$ is about of $3 \times 10^{-7} \mathrm{~m}$, which is much less than the typical sample layer thickness $L \approx 10^{-4} \mathrm{~m}$.

The total sample absorptivity depends on the concentration of the solute as follows:

$$
\alpha=\alpha_{0}+\varepsilon(\lambda) C,
$$

where $\alpha_{0}$ is the absorptivity of the pure solvent, $\varepsilon(\lambda)$ is the molar absorptivity of the solute depending upon the laser beam wavelength $\lambda$.

OA measurements are conducted with the use of socalled optoacoustic cells. Their design varies according to this or that task. The problem of OA measurements here consists in establishing a link between the parameters of acoustical disturbance according to (1) and the concentration of a solute, which affects the absorptivity defined from (2). The aim of the paper is to calculate the parameters of elastic response in a typical optoacoustic 
cell for small-volume samples and to estimate the figures of merit of this version of calorimetric spectroscopy.

\section{Optoacoustic measurement cells}

Figure 1 shows two popular types of the optoacoustic cells, which have been used for the measurements in the liquid samples of a relatively large volume, 10-100 ml. Lateral optoacoustic cell (Fig. 1a) has found wide application since the late seventies $[1,2]$. The cell has inlet and outlet windows. Laser beam 1 enters and leaves the cell through the windows. Laser energy is partially absorbed in the sample 2. The thermalized energy is sufficient for the excitation of the acoustic response. In turn, acoustic pulse is recorded by a sound pressure transducer 3 in the field of a cylindrical divergent wave. The transducer is attached to the backing damper 4. Output signal is fed to the processing system. Usually, lateral cell provides the magnitude of acoustic response in a relatively low-frequency band (up to $1 \mathrm{MHz}$ ). The cell of the second type, namely, front face (FF) cell (Fig. 1b), has been designed for time-resolving optoacoustic measurements. Such a cell is more sensitive as compared to the previous type. FF cell assumes more complete utilization of the acoustical spectrum. Here, beam 1 enters FF cell through the window, propagates over the liquid sample and is reflected from a mirror 5 , which protects the transducer. The outgoing elastic pulse from the sample passes the mirror and excites the transducer 3 attached to the backing damper. FF cells were used for the dynamic measurements of the parameters of liquid samples with sub-nanosecond time resolution $[5,6]$. The design of both cells makes it possible to record signals in the near acoustic field. Such signals are convenient for the interpretation, which in turn, helps to restore the parameters of the sample. However, the attempts to use these cells for the analysis of small-volume samples were not very successful: the reverberation interference caused by the vicinity of various interfaces substantially grew up. The direct transducer exposure with the scattered laser beam was the second source of high-level noise. The sensitivity of measurements thus reduced significantly.
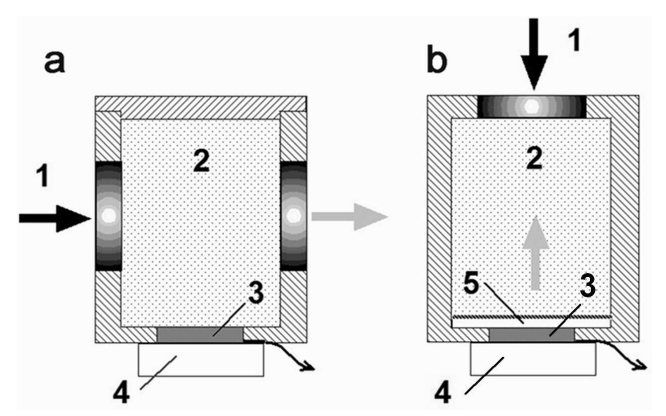

Fig. 1. Optoacoustic cells for relatively large volume samples. (a) Lateral cell, (b) front face cell. 1 - incoming light pulse, 2 - sample, 3 - sensor, 4 - backing dumper, 5 - mirror.
It appeared that optoacoustic measurements of smallvolume liquid samples can show good sensitivity when conducted with the help of a so-called layered prism (LP) cell, see Fig. 2a. Laser beam 1 excites the initial pressure increment in the thin sample layer 2, clamped between the upper and lower glass prisms. The direct light exposition of the transducer is excluded due to the inclined trajectory of laser beam. For the free propagation of the incoming laser beam, it is necessary to provide that the thickness of the lower prism is not less than $0.02 \mathrm{~m}$. The thickness of the sample layer $L$ is defined by the thickness of a shim 5 being changed within a range $10^{-5}$ to $45 \times 10^{-5} \mathrm{~m}$.
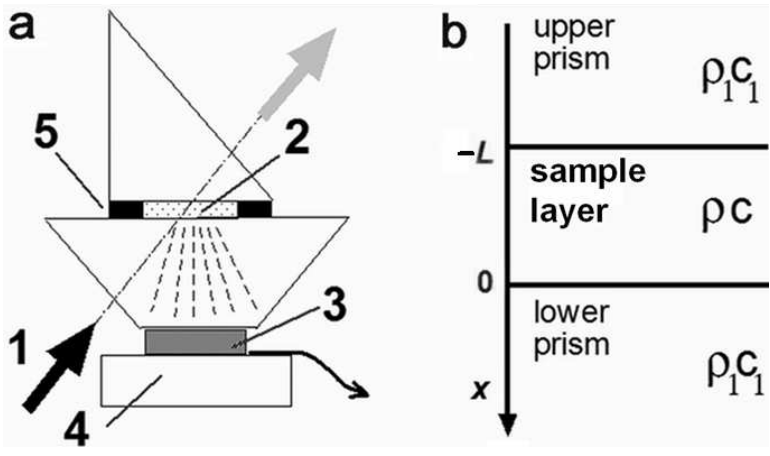

Fig. 2. Layered prism optoacoustic cell. 1 - incoming light pulse, 2 - sample, 3 - sensor, 4 - backing dumper, 5 - shim. (b) Simplified model scheme.

Outgoing elastic pulse (dashed lines) propagates from the sample layer towards the pressure transducer 3 . The propagation path length substantially exceeds the same for the previously described cells. The pick-up of the signal is carried out in the transitional or even in the far-field zone of the source. While propagating, the profile of the signal can noticeably evolve, and its interpretation can be hindered. This is the price for the possibility to conduct measurements in small-volume samples with no high-level interference. It is important that the sample layer 2 and the transducer 3 are arranged in parallel to each other. The LP cells have been in use since the nineties [7].

\section{Generation and recording of elastic pulse with the use of LP cell}

Phenomenologically, measurement process with the use of LP cell can be described as follows (see Fig. 2b). Relaxation of initial pressure increment having the magnitude $p_{0}(1)$ leads to the excitation of elastic pulses, propagating through both upper and lower prisms. The pulse propagating through the lower prism along the axis $x$ is of the most interest. It presents a useful informative signal. On entering the lower prism the signal belongs to the near-field wave zone. Then the pulse is of a unipolar form, its typical width being determined by the thickness of the sample layer $L$ along with the acoustical 
transmission and reflection coefficients at the "sampleglass" interface. In turn, these coefficients depend on the relationship of the acoustic impedances $\rho c$ and $\rho_{1} c_{1}$. The pulse profile contains the periodic structures (steps), since the sample layer presents a high-quality acoustic resonator. The near-field magnitude of the elastic pulse approximately equals $\sigma_{0}=p_{0} W / 2$, where $W$ is the acoustical transmission coefficient, $W=2 \rho_{1} c_{1} /\left(\rho_{1} c_{1}+\rho c\right)$. During the pulse propagation through the lower prism, diffraction evolution of the profile occurs. Piezoceramic pressure transducer responds to the arrival of the pulse in a specific manner. The undistorted time-resolving pick-up of the pulse is possible provided the design of both the transducer and the processing system is done in a proper way.

The closer approximation to the description of the whole process is possible by means of a step-by-step approach with the use of a transfer functions method [8]. The spectrum of the elastic pulse $\sigma(\omega, x)$ at a distance $x$ from "sample-glass" interface is proportional to the product of the laser pulse pressure spectrum $F(\omega)$ and the two specific transfer functions,

$$
\sigma(\omega, x) \propto F(\omega) G(\omega) D(\omega, x),
$$

where $G(\omega)$ is a transfer function, which describes the generation of the pressure transients in the sample and $D(\omega, x)$ is a transfer function, which describes the diffraction transformation of the elastic signal in the course of its propagation through the lower prism along the axis $x$. Then, we can put down for an elastic pulse in the time domain

$$
\begin{aligned}
& \sigma(t, x)=\mathrm{i} a^{2} \frac{\beta \alpha}{2 C_{p} x} \int F(\omega) G(\omega) D(\omega, x) \\
& \quad \times \exp \left(\mathrm{i} \omega\left(t-x / c_{1}\right)\right) \mathrm{d} \omega .
\end{aligned}
$$

An expression for laser pulse envelope spectrum $F(\omega)$ depends on this or that specific case. For example, for a laser pulse of a triangular form with the base width $\tau$ and the peak intensity $I_{0}$ the expression takes the form

$$
F(\omega)=I_{0} \tau[1-\cos (\omega \tau / 2)] /(\omega \tau / 2)^{2} .
$$

Considering the liquid sample layer having thickness $L$ clamped between the two prisms of the same glass material, one can put down for $G(\omega)$, in view of the works [6] and $[9]$ :

$$
\begin{aligned}
& G(\omega)=\frac{1}{2} \rho c \frac{W}{i \omega} \\
& \times\left(\frac{1-\left(\rho c / \rho_{1} c_{1}\right) W \exp (-\mathrm{i} k L)-V \exp (-\mathrm{i} 2 k L)}{1-V^{2} \exp (-\mathrm{i} 2 k L)}\right),
\end{aligned}
$$

where $V$ is the acoustical reflection coefficient at the "sample-glass" interface, $V=\left(\rho_{1} c_{1}-\rho c\right) /\left(\rho_{1} c_{1}+\rho c\right)$, $k$ is the wave number. The above expression allows more simple presentation

$$
G(\omega)=\frac{1}{2} \rho c L \frac{\sin (k L / 2)}{k L / 2} \frac{W \exp (-\mathrm{i} k L / 2)}{1-V \exp (-\mathrm{i} k L)} .
$$

For the case of a Gaussian magnitude distribution over cross-section of an elastic beam propagating in the lower prism, we can put down for $D(\omega, x)$ :

$$
D(\omega, x) \propto \frac{\omega}{1+\mathrm{i} 2 \pi a^{2} \omega / 2 x c_{1}} .
$$

The expression (4) along with (5)-(8) served as a basis for MatLab modeling of the outgoing elastic signal. As a result, Fig. 3 shows the signal evolution while traveling from the sample to the transducer through the lower prism along the normal. In Fig. 3a there is given the signal at a distance $x=0.001 \mathrm{~m}$ from the upper edge of lower prism, Fig. 3b corresponds to a distance $x=0.012 \mathrm{~m}$, and Fig. 3c corresponds to a distance $x_{\mathrm{t}}=0.02 \mathrm{~m}$, corresponding to the entrance of the transducer. One can estimate the magnitude of the elastic pulse at any distance from the sample layer, using an expression $\sigma_{0, x}=M(x) \sigma_{0}$, where $M(x)<1$ at $x>0$. The magnitude factor $M(x)$ can be evaluated directly from (4). Its value is marked with arrows in Fig. 3. Having partially undergone the diffraction at the propagation stage the signal obtains bi-polar shape. The periodic structures which can be seen in the curves, have a period close to $L / c$ and occur due to the fact that a sample forms a high-quality resonator.

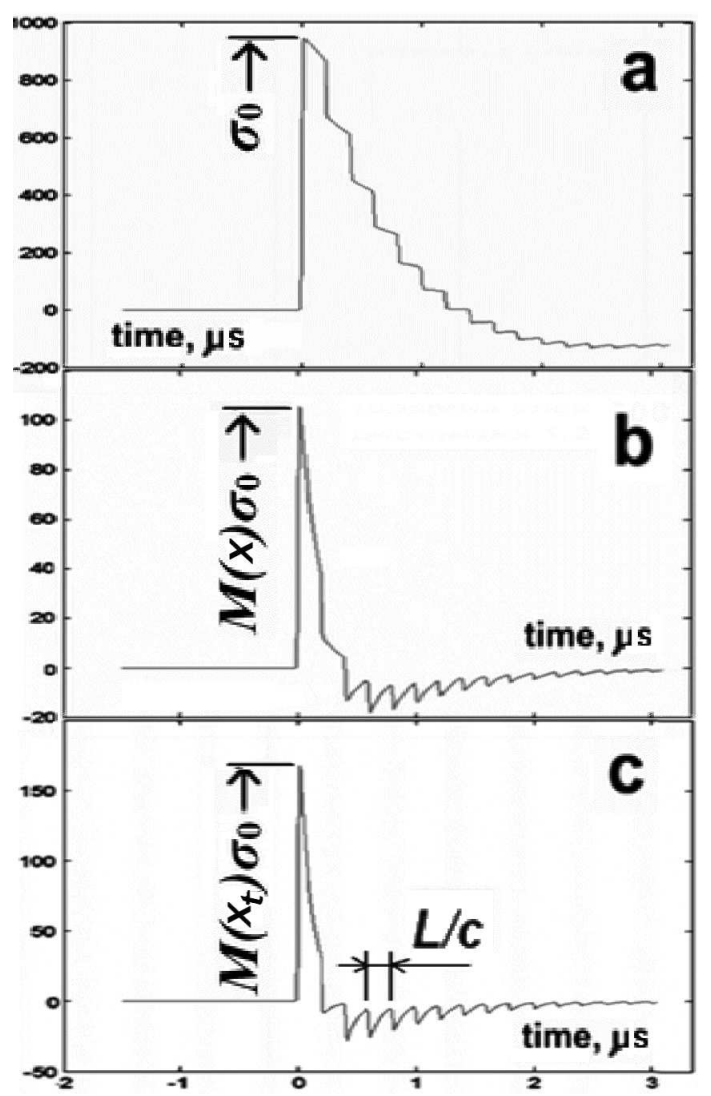

Fig. 3. Evolution of the elastic pulse profile in the lower prism. MatLab based modeling. $L=0.0003 \mathrm{~m}$, $a=0.002 \mathrm{~m}$. (a) The pulse enters the lower prism, (b) in the middle of the distance, (c) the pulse enters the transducer. Vertical scale: mechanical stress (arbitrary units).

Consider now the resulting electric signal $U$, which is fed to a load having impedance $Z_{\mathrm{E}}$ as an output of the piezoceramic transducer excited by the incoming elastic 
pulse. We use a transfer function $S(\omega)$ (or spectral sensitivity in other words), $S(\omega)=U(\omega) / \sigma\left(\omega, x_{\mathrm{t}}\right)$, linking the electric voltage $U(\omega)$ on the load $Z_{\mathrm{E}}(\omega)$ to the incoming elastic pulse $\sigma\left(\omega, x_{\mathrm{t}}\right)$ in the frequency domain. For the case of short incoming elastic pulses, expressions for $S(\omega)$ were considered in [10].

The best case corresponds to an active load $Z_{\mathrm{E}}(\omega)=$ $R$, while the spectrum of the incoming elastic pulse is restricted to the following limits, $\omega \tau_{\mathrm{p}} \ll 1$ and $\omega \tau_{\mathrm{RC}} \gg 1$, where $\tau_{\mathrm{RC}}=\mathrm{RC}_{\mathrm{s}}$ is the time constant defined by the capacitance $C_{\mathrm{S}}$ of the transducer and the resistance of the load $R, \tau_{\mathrm{p}}$ is the travel time of elastic pulse across the thickness of the piezoceramic transducer plate. It can be shown that for this case in view of [10] we can put down for the spectral sensitivity

$$
S(\omega)=\frac{h_{33} W_{f} \tau_{\mathrm{p}}}{Z_{2}} .
$$

Here $Z_{2}=\rho_{2} c_{2}$ is the acoustic impedance of the piezoceramic transducer plate, $W_{f}$ is the elastic transmission coefficient at the "glass-ceramics" interface, $\rho_{2}$ is the density, $c_{2}$ is the sound velocity, $h_{33}$ is the piezoelectric modulus of the transducer material. For a simple form profile of the elastic pulse, the above mentioned conditions obtain a simple physical meaning. Indeed, the signal spectrum mostly belongs to the frequency value $1 / \tau_{\mathrm{a}}$, where $\tau_{\mathrm{a}}$ is the specific width of an acoustic pulse. The aforecited inequalities thus take the form: $\tau_{\mathrm{p}} \ll \tau_{\mathrm{a}}, \tau_{\mathrm{RC}} \gg \tau_{\mathrm{a}}$. This means that an acoustically thin piezoceramic transducer is used and the time constant $\tau_{\mathrm{RC}}$ is large as compared to the acoustic pulse width.

Therefore, the sensitivity (9) is a constant value, independent of frequency, $S(\omega)=S$. Thus, the above mentioned conditions turn to be the conditions of the pure signal recording with an appropriate time resolution. Furthermore, the sensitivity value is proportional to the transducer's thickness. One can make the following estimate for $S$. For the transducer thickness $4 \times 10^{-4} \mathrm{~m}, \rho_{2}=7.5 \times 10^{3} \mathrm{~kg} / \mathrm{m}^{3}, c_{2}=4200 \mathrm{~m} / \mathrm{s}$ and $h_{33}=0.2 \times 10^{10} \mathrm{C} / \mathrm{N}$, we get $S=6 \mu \mathrm{V} / \mathrm{Pa}$. Here, we assume that the "glass-ceramics" interface is acoustically matched and $T_{f}=1$. Hence we defined possible parameters of an optimized LP cell.

\section{OA measurements with the use of LP cell: figures of merit}

As an example of the measurement process applying optimized LP cell, let us evaluate sensitivity of the reagentless determination of lipopolysaccharides (LPS) as the most infamous class of pyrogenic substances. For the given LP cell design, the limit of detection (LOD) of LPS is calculated according to the IUPAC-1998 recommendations for presentation of the results of chemical analysis.

We should use a frequently used OA measurement parameter, namely optoacoustic responsivity $Y$ defined as a ratio of the useful voltage response magnitude to the specific thermalized energy, $Y=U_{\mathrm{m}} / \alpha E_{0}$. For the case of LP cell configuration, in view of Eqs. (3)-(9), the following expression is valid, $Y=S M\left(x_{\mathrm{t}}\right) W \beta c^{2} / 2 \pi a^{2} C_{\mathrm{p}}$.

We restrict ourselves to the magnitude version of OA measurement method. With respect to the previously introduced variables optoacoustic spectroscopy equation for receiver output magnitude can be put down as follows:

$$
U_{\mathrm{m}}=N \varepsilon(\lambda) C E_{0} Y+N \alpha E_{0} Y+u_{\mathrm{N}} \sqrt{N}
$$

where $u_{\mathrm{N}}$ is the output noise level of the receiver and $N$ is the number of shots to be averaged. Here, the second addend represents the background contribution of the pure solvent. With $C \rightarrow 0$ expression (10) yields a simple relation for the limiting detectable absorptivity of the solvent

$$
\alpha_{\min }=u_{\mathrm{N}} /\left(E_{0} Y \sqrt{N}\right) .
$$

The value $\alpha_{\text {min }}$ so defined is truly inversely proportional to the pulse energy. Substituting $E_{0}=0.3 \mathrm{~mJ}$, $N=1000$ along with the other parameters typical for LP cell in (11), we get $\alpha_{\min } \approx 10^{-4} \mathrm{~m}^{-1}$. It can be shown that the LOD of the solute $C_{\min , \mathrm{P}}$ is introduced as follows according to IUPAC rules in our case

$$
C_{\text {min }, \mathrm{P}}=t_{p, f} S_{0} / \varepsilon(\lambda),
$$

where $P$ is the probability of detection, $t_{p, f}$ is the Student coefficient, $f$ is the number of degrees of freedom, $f=m-2, S_{0}$ is the absolute error of the pure solvent response compensation, $m=16$ is the number of calibration tests. For the case of LPS determination one can assume that $\varepsilon(\lambda)=10$ liter $/$ mole $/ \mathrm{m}$ [11]. Substituting necessary values in (12), we get $S_{0} \approx \alpha_{\min } \approx 10^{-4} \mathrm{~m}^{-1}$. The technical issues of such compensation procedure are out of the scope of this paper.

Equation (12) shows that the determination of LPS with the use of the LP cell can be of better quality than that achieved by our team earlier with the use of a lateral OA cell [11]. In the present case, one can provide LOD below the level of $0.1 \mu \mathrm{g} / \mathrm{ml}$, which is a rather good sensitivity for chemical methods for LPS and is comparable to that of the state-of-the-art biological test for LPS, namely, limulus amoebocyte lysate test (LAL) [12].

\section{Conclusion}

Optoacoustic measurement process with the use of the layered prism cell was described within a step-by-step approach based on the transfer functions method. Our results indicate that this cell provides sufficient figures of merit of determination of the low-concentration impurities in small-volume liquid samples. The layered prism cell is superior to the cells of early design for achieving the lowest possible limits of detection of various impurities. In summary, the results make a contribution to further development of optoacoustic methods and equipment to be applied for chemical analysis.

\section{Acknowledgments}

The author wishes to thank Dr. Andrey Fokin for useful discussions. 


\section{References}

[1] J.I. Kim, R. Stumpe, R. Klenze, Topics Curr. Chem. 157, 129 (1990).

[2] A.C. Tam, in: Photoacoustic and Photothermal Phenomena III, Ed. D. Bicanic, Springer-Verlag, Berlin 1992, p. 447.

[3] S.V. Egerev, L.M. Lyamshev, A.E. Pashin, J. Phys. IV (France) Coll. Cr Suppl. III 4, C7 (1994).

[4] S. Egerev, O. Ovchinnikov, AIP Conf. Proc. 1433, 729 (2012).

[5] L.G. Arnaut, R.A. Caldwell, J.E. Elbert, L.A. Melton, Rev. Sci. Instrum. 63, 5381 (1992).

[6] O. Puchenkov, S. Malkin, Rev. Sci. Instrum. 67, 672 (1996).
[7] T. Autrey, N. Foster, K. Klepzig, J. Photochem. Photobiol. A Chem. 125, 13 (1999).

[8] V.E. Gusev, A.A. Karabutov, Laser Optoacoustics, American Institute of Physics, New York 1993.

[9] M.I. Khan, T. Sun, G.J. Diebold, J. Acoust. Soc. Am. 93, 1417 (1993).

[10] G. Hayward, C.J. MacLeod, T.S. Durrani, J. Acoust. Soc. Am. 76, 369 (1984).

[11] M. Proskurnin, E. Ageeva, V. Senyuta, N. Orlova, S. Egerev, Talanta 81, 377 (2010).

[12] K.L. Williams, Endotoxins: Pyrogens, LAL Testing, and Depyrogenation, Marcel Dekker, New York 2001. 